\title{
Hybrid Segmentation of Vessels and Automated Flow Measures in In-Vivo Ultrasound Imaging
}

Moshavegh, Ramin; Martins, Bo; Hansen, Kristoffer Lindskov; Bechsgaard, Thor ; Bachmann Nielsen, Michael; Jensen, Jørgen Arendt

\section{Published in:}

Proceedings of 2016 IEEE International Ultrasonics Symposium

\section{Publication date:}

2016

Document Version

Peer reviewed version

Link back to DTU Orbit

Citation (APA):

Moshavegh, R., Martins, B., Hansen, K. L., Bechsgaard, T., Bachmann Nielsen, M., \& Jensen, J. A. (2016). Hybrid Segmentation of Vessels and Automated Flow Measures in In-Vivo Ultrasound Imaging. In Proceedings of 2016 IEEE International Ultrasonics Symposium IEEE.

\section{General rights}

Copyright and moral rights for the publications made accessible in the public portal are retained by the authors and/or other copyright owners and it is a condition of accessing publications that users recognise and abide by the legal requirements associated with these rights.

- Users may download and print one copy of any publication from the public portal for the purpose of private study or research.

- You may not further distribute the material or use it for any profit-making activity or commercial gain

- You may freely distribute the URL identifying the publication in the public portal 


\title{
Hybrid Segmentation of Vessels and Automated Flow Measures in In-Vivo Ultrasound Imaging
}

\author{
Ramin Moshavegh*, Bo Martins ${ }^{\ddagger}$, Kristoffer Lindskov Hansen ${ }^{\dagger}$, \\ Thor Bechsgaard ${ }^{\dagger}$, Michael Bachmann Nielsen ${ }^{\dagger}$ and Jørgen Arendt Jensen* \\ ${ }^{*}$ Center for Fast Ultrasound Imaging, Dept. of Elec. Eng., Technical University of Denmark, Lyngby, Denmark \\ ${ }^{\dagger}$ Dept. of Radiology, Copenhagen University Hospital, DK-2100 Copenhagen, \\ Denmark, ${ }^{\ddagger}$ BK Ultrasound ApS, Herlev, Denmark
}

\begin{abstract}
Vector Flow Imaging (VFI) has received an increasing attention in the scientific field of ultrasound, as it enables angle independent visualization of blood flow. VFI can be used in volume flow estimation, but a vessel segmentation is needed to make it fully automatic. A novel vessel segmentation procedure is crucial for wall-to-wall visualization, automation of adjustments, and quantification of flow in state-of-the-art ultrasound scanners. We propose and discuss a method for accurate vessel segmentation that fuses VFI data and B-mode for robustly detecting and delineating vessels. The proposed method implements automated VFI flow measures such as peak systolic velocity (PSV) and volume flow. An evaluation of the performance of the segmentation algorithm relative to expert manual segmentation of 60 frames randomly chosen from 6 ultrasound sequences (10 frame randomly chosen from each sequence) is also presented. Dice coefficient denoting the similarity between segmentations is used for the evaluation. The coefficient ranges between 0 and 1 , where 1 indicates perfect agreement and 0 indicates no agreement. The Dice coefficient was 0.91 indicating to a very agreement between automated and manual expert segmentations. The flowrig results also demonstrated that the PSVs measured from VFI had a mean relative error of $14.5 \%$ in comparison with the actual PSVs. The error for the PSVs measured from spectral Doppler was $29.5 \%$, indicating that VFI is $\mathbf{1 5 \%}$ more precise than spectral Doppler in PSV measurement.
\end{abstract}

\section{INTRODUCTION}

Accurate delineation of vessels is crucial for visualization and quantification of flow in state-of-the-art ultrasound scanners. Available segmentation procedures are mainly based on either B-mode images or flow estimates only. However, the main challenge in devising an accurate vessel segmentation procedure is to incorporate both B-mode image and flow data for better identifying the vessels. This paper presents a hybrid segmentation algorithm that fuses B-mode and vector velocity estimations (VFI) for robustly detecting and segmenting vessels in ultrasound images. VFI enables angle independent visualization of blood flow, and allows doctors to see and estimate the direction and velocity of blood flow in all directions and at any angle. This provides an angle independent visualization of blood hemodynamics for the cardiovascular system in real time [1]. Today's commercial ultrasound scanners are not able to present a perfect wall-towall delineation of vessels, while displaying the VFI images.
Of particular interest is the extraction of new features that can express several desired properties of a vessel (i.e. constriction in carotid artery) in a single feature such as peak systolic velocity (PSV) [2], [3] using VFI. The volume flow is also a very interesting measure when inspecting the VFI, which quantifies the flow and characterizes the vessel [4], [5], [6], [7]. However, this measure is very susceptible to the vessel diameter, which makes the accurate delineation of the vessel very essential [6], [8]. The proposed method delineates the vessel walls and enables the correct estimation of the volume flow. In the current implementation of VFI in ultrasound scanners, the correct volume flow estimation is not possible. The reason is that the VFI does not perfectly attach to the vessel walls, and therefore the VFI data is not available for regions very close to the vessel walls. This paper proposes a novel hybrid vessel segmentation in ultrasound that also enables the automated flow quantification. The proposed method delineates the vessels in ultrasound scans and enables a better visualization of flow inside the vessel, as well as providing the firm ground for quantitative flow measures for VFI such as PSV and volume flow. These are done without manual adjustments by users and without compromising on the accuracy of VFI. The remainder of this paper is organized as follows. Section II introduces the algorithm. Section III presents the measurement setup. Section IV presents the results and discusses the findings. Finally section $\mathrm{V}$ is the conclusion.

\section{Materials AND Methods}

The method has two distinct steps. First, it automatically segments the vessel boundaries using a combination of VFI data and B-mode. Second, it automates the VFI flow measures such as PSV, and volume flow.

\section{A. Novel hybrid segmentation of vessels}

The segmentation has four distinct steps. First, a marker image is generated from both VFI data and B-mode. An example of a marker image computed for a carotid phantom scan with bifurcation is shown in Fig. 1(f). Second, the marker image is used in a subsequent marker-controlled region growing procedure to delineate the vessel boundaries. The third 


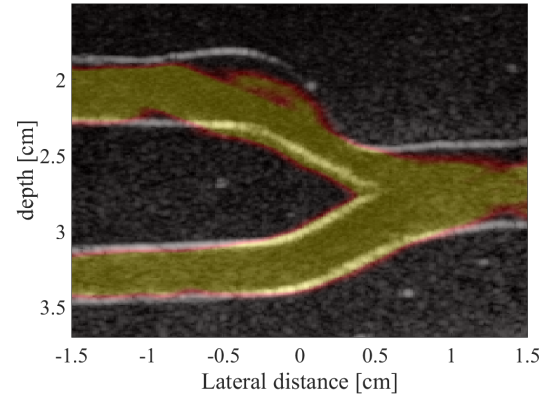

(a) Absolute value of VFI overlaid on B-mode.

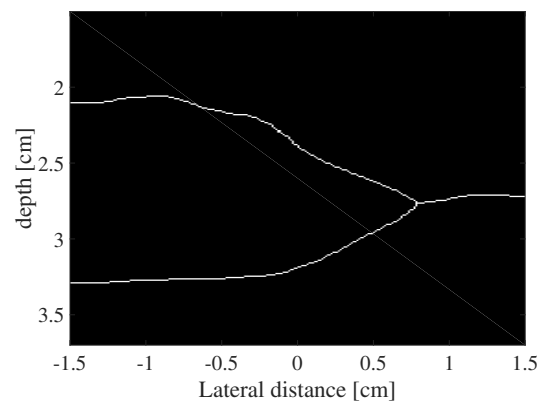

(d) Center-line of image in (c) (inner markers)

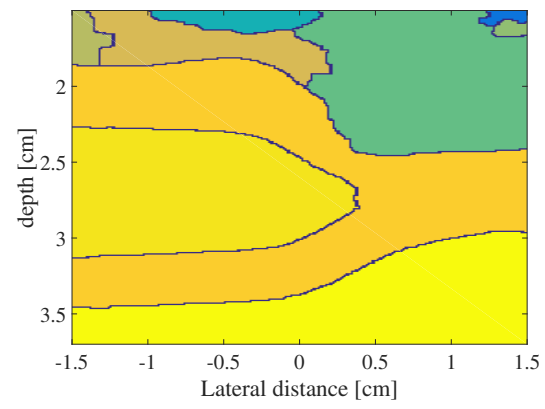

(g) Result of watershed segmentation.

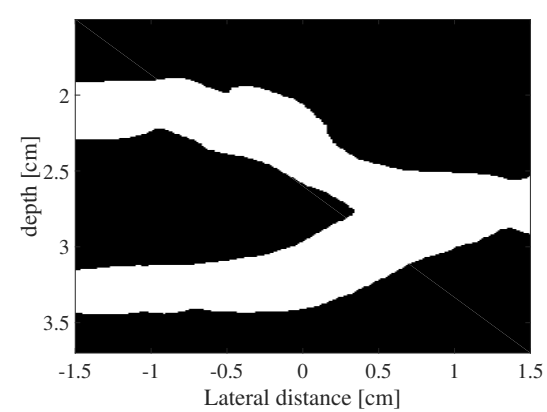

(b) Binary mask of VFI data.

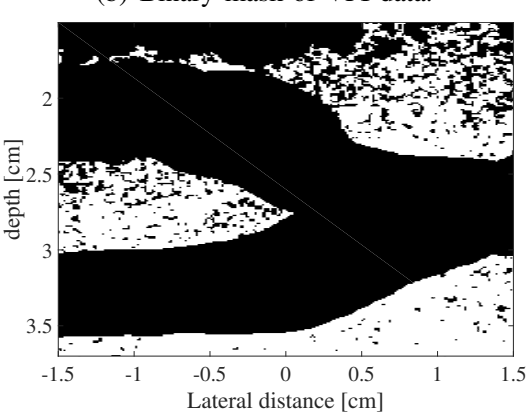

(e) Outer markers generated from B-mode.

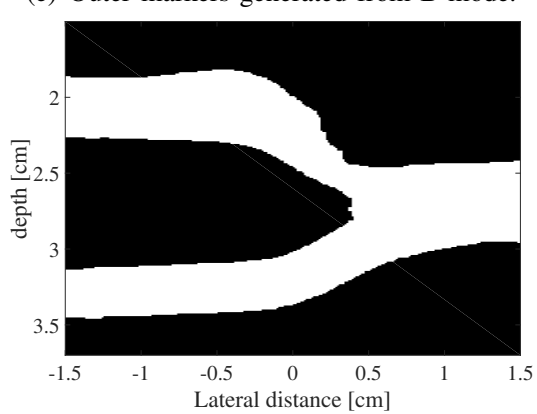

(h) Artifacts rejected from image $(g)$.

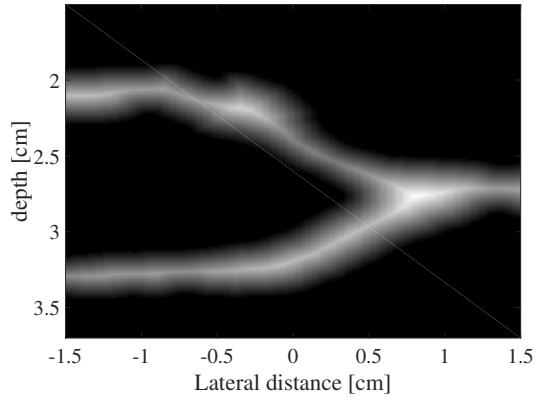

(c) Distance transform of inverted mask in $(b)$.

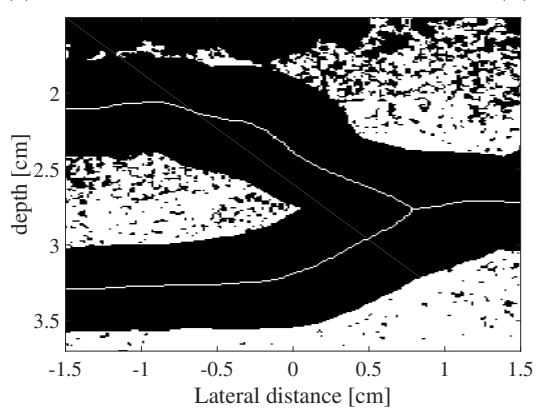

(f) Marker image -combination of $(d)$ and $(e)$.

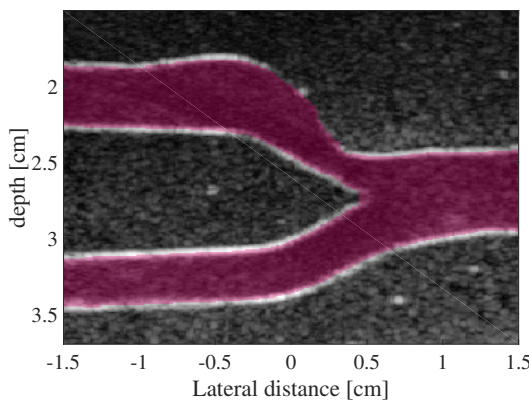

(i) Inter-frame co-registration and refinement.

Fig. 1. Illustration of segmentation performed on a sequence from a carotid phantom with a bifurcation.

step implements artifact rejection based on size, shape, and amount of flow to ensure that only vessels are retained. Fourth step implements an inter-frame co-registration of consequent segmented frames to make sure that vessels segmented in each frame are in correspondence with the same vessels segmented in the neighboring frames of the sequence.

\section{1) Generating the marker image:}

Extraction of inner-markers: Detection of inner markers are achieved by skeletonization that extracts the center-line of the VFI data. The absolute value of VFI estimates were used in segmentation procedure. The process extracted the centerline of the flow data that also indicated the locations inside the vessel boundary. The skeletonization started by computing the distance transform of the inverted binary mask of VFI data (see Fig. 1(b) and 1(c)). Then the ridge lines on the distance transform are considered as the center-line of the flow data (see Fig. 1(d)).

Extraction of outer-marker mask: The detection of outer markers are achieved using the B-mode image. Strong specular regions are extracted from the B-mode and used as an indication of regions outside the vessels. For this purpose, the envelope of the acquired scan is first computed. A cumulative histogram of the intensity values is then generated. The intensity values less than half of the maximum intensities in the envelope are disregarded, and a mask of strong signal regions is generated. The mask is then multiplied to a dilated version of the VFI mask and yielded the outer marker mask as shown in Fig. 1(e). The inner and outer markers are combined in one marker-image binary mask (see Fig. 1(f)), and used to initialize the region growing algorithm on the B-mode image.

2) Marker-controlled region growing:

Marker-controlled watershed is used to delineate the boundaries of the vessel-like structures bracketed by the marker image. The B-mode image is median filtered, and a watershed segmentation of this filtered image with respect to the Marker image yielded the segmentation of the vessel-like structures (see Fig. 1(g)). However, segmentation results contain nonvessel structures that should be excluded.

\section{3) Artifact rejection:}

The region growing resulted in over-segmented regions, which are not vessels. This step implements artifact rejection 


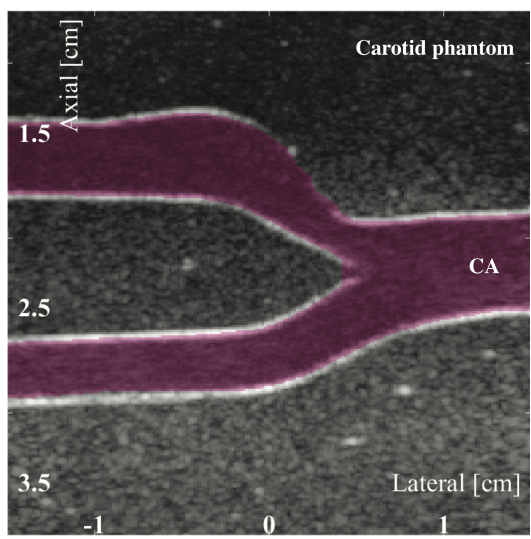

(a) Carotid artery segmented on carotid phantom.

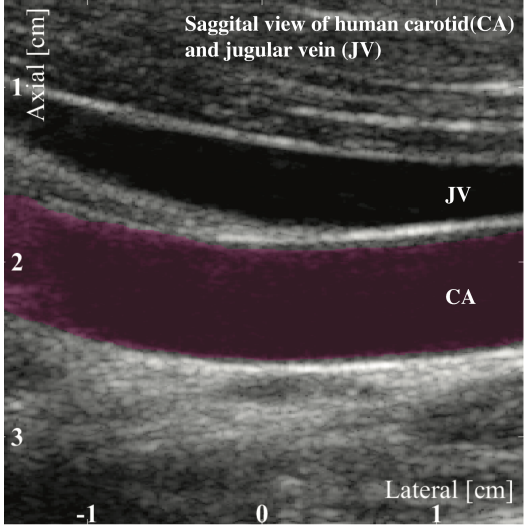

(b) Carotid artery segmentation In-Vivo.

Fig. 2. Results of carotid segmentations performed on phantom and in-vivo scans.

based on size, shape and amount of flow to ensure that only the vessels are retained(see Fig.1(h)). Basically, elongated objects containing flow data are kept in segmentation results and the rest are excluded. Even though, the retained structures in this step are vessel-like objects, the boundaries of the vessels might have some discrepancies with respect to the actual vessel boundaries.

4) Inter-frame co-registration of segmentations:

To refine the segmentation, so that it follows the exact vessel boundaries, an inter-frame co-registration of segmentations is performed. To deform parts of the segmentation that does not follow the actual vessel boundaries, 10 to 25 frames (according to the frame rate) neighboring in time are considered. Segmentations performed on all the neighboring frames are coregistered and regions matching in at least $80 \%$ of the frames are considered to be the actual vessels (See Fig. 1(i)).

\section{B. Enabling VFI automated measures}

In this section the possibility of automating the two crucial flow measures such as PSV and volume flow using VFI are introduced.

1) Automated Peak systolic velocity (PSV) measurement using VFI:

Using the proposed method, the vessel walls are accurately delineated. Fig. 5 shows an example of segmentation of a human carotid. The red lines indicate the location of the vessel walls delineated by the proposed method. Two points are set across the vessel on the delineated vessel boundaries. Then, PSV along the line connecting the two points, through the vessel, across a cardiac cycle is calculated (see Fig. 5). The red horizontal line indicates the position as well as the average peak velocity angle throughout the cycle. The green horizontal line indicates the center and the circular cross section found inside the vessel for measuring the volume flow.

2) Automated volume flow measurement using VFI:

First, the vessel walls are automatically delineated, and VFI is refined so that it covers the whole vessel region. This enables the correct estimation of volume flow. Second, the volume flow can be computed without user manual intervention of a user. This is performed by automatically selecting vessel cross-sections between the segmented walls for computing the volume flow in VFI (see yellow circular cross section in Fig. 5). The volume flow is implemented using the method proposed in [8].

\section{Measurement SETUP}

The proposed segmentation algorithm was applied to 5 carotid artery sequences. Five healthy subjects were scanned, and a total of 5 carotid artery sequences each containing 500 frames were acquired. Also a phantom including a carotid with a bifurcation was scanned and used in this study. The beamformed RF data were acquired using a BK3000 scanner (BK Ultrasound, Denmark) connected to a 192-element 4.1 $\mathrm{MHz}$ linear array transducer (9032, BK Ultrasound). The beamformed RF data were simultaneously recorded by a research interface on the scanner, and processed with the proposed algorithm off-line. For in-vitro flowrig validation of VFI, a flow system (CompuFlow 1000, Shelley Medical Imaging Technologies, Toronto, Canada) circulating a bloodmimicking fluid (BMF-US, Shelley Medical Imaging Technologies, Toronto, Canada) in a closed loop circuit was used. The linear transducer was fixed at a distance of $1.5 \mathrm{~cm}$ from the vessel of $12 \mathrm{~mm}$ in diameter with a beam-to-flow angle of 60 degrees. For increasing constant flowrig peak velocities of 5-60 cm/s, Spectral Doppler and VFI data were recorded. For precision analysis, each velocity setting was recorded twice. All the measures are implemented in a in-house application specialized for VFI visualization and quantitative flow measures. All the PSV and volume flow measurements are performed using this application. The distribution of peak velocity amplitudes and angles during one cardiac cycle are also automatically computed and reported in the tool.

\section{RESULTS AND DISCUSSION}

Automated segmentation results were compared with manual segmentations performed by an experienced user on 60 frames (10 frame randomly chosen from each sequence). Dice coefficient denoting the similarity between segmentations was 


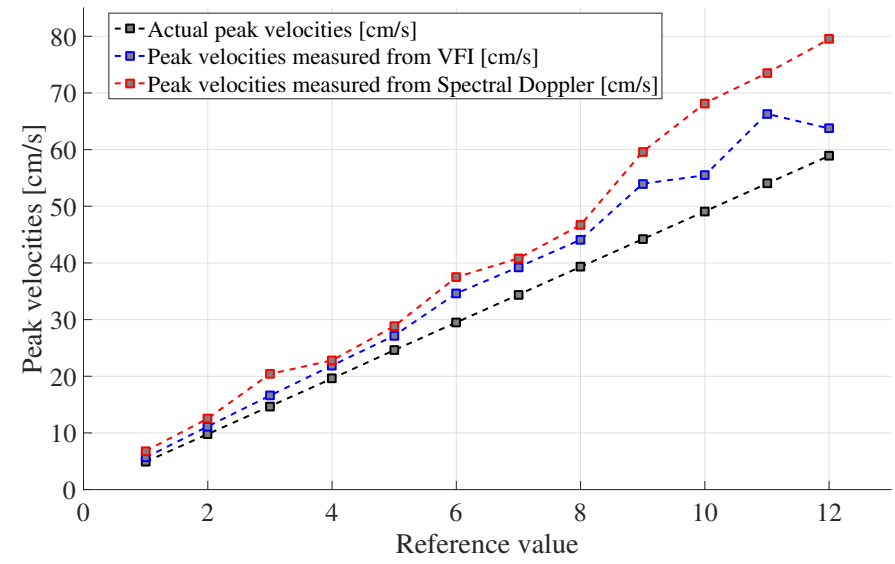

Fig. 3. Comparison of flowrig PSV measured by VFI and spectral Doppler.

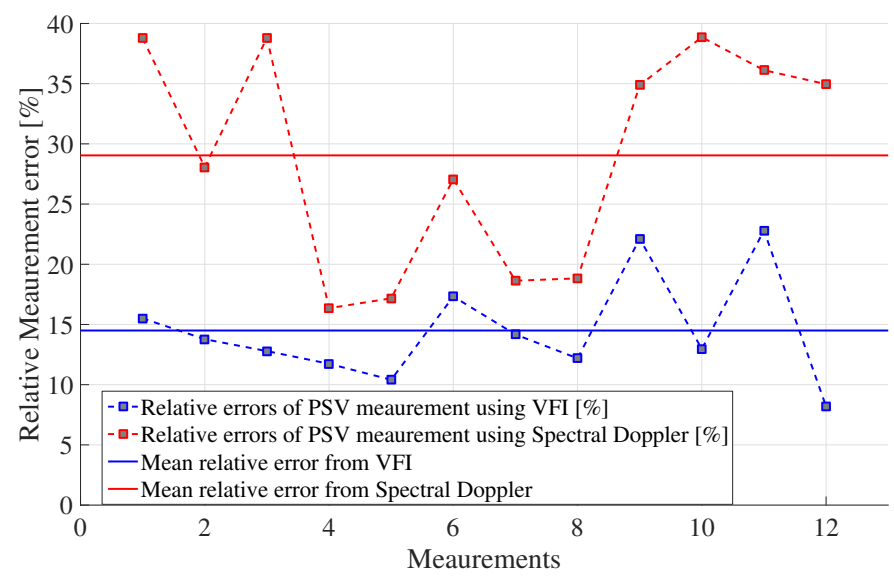

Fig. 4. Relative errors of measurements with VFI and spectral Doppler.

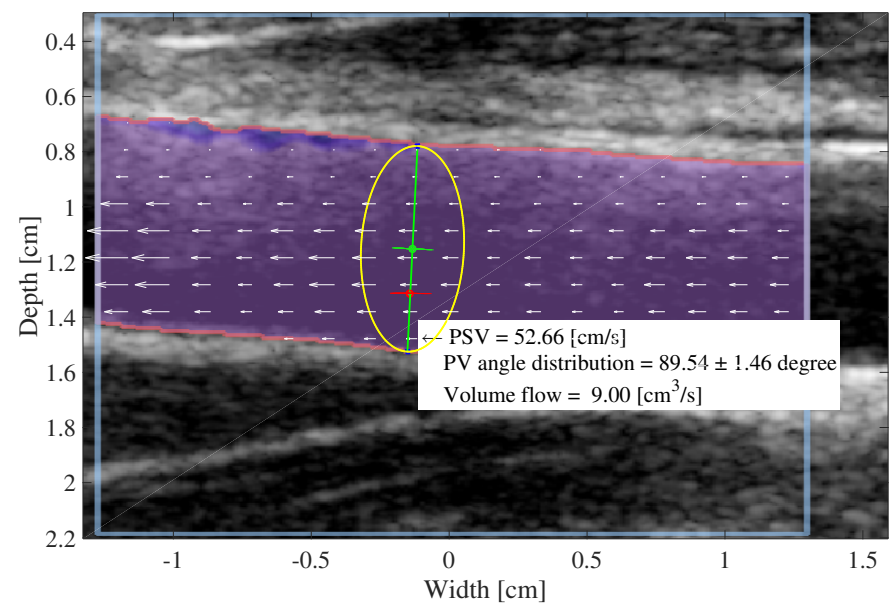

Fig. 5. Carotid artery segmented, The VFI is visualized, and a cross-section is automatically selected to compute the PSV and volume flow.

then computed. The coefficient ranges between 0 and 1 . A value of 1 indicates perfect agreement and a value of 0 indicates no agreement. The Dice coefficient was 0.91, indicating that vessel boundaries obtained using the algorithm are highly accurate and consistent with the experts' visual perception of vessel boundaries. Two examples of segmentations performed by the proposed hybrid method on scans acquired from a carotid phantom with a bifurcation and carotid of a normal male subject are shown in Figs. 2(a) and 2(b) respectively. Fig. 3 shows the results of in-vitro flowrig validation of VFI for measuring PSV. Relative errors of the PSVs measured from VFI and spectral Doppler comparing to the actual PSVs were $14.5 \%$ and $29.5 \%$ respectively (see Fig. 4). The figure shows that VFI offers a more precise and reliable alternative for velocity estimation of PSV in comparison with the current clinical standard using spectral Doppler. Fig. 5 shows a screen shot of the CFU visualization application, in which a carotid artery belonging to a 29 years old healthy male subject is segmented and VFI is visualized. The PSV and volume flow are also measured using the application and reported. PSV was $52.66 \mathrm{~cm} / \mathrm{s}$ and the volume flow was $9 \mathrm{~cm}^{3} / \mathrm{s}$. The distribution of angle during one cardiac cycle was $89.54 \pm 1.46$ degrees.

\section{CONClusion}

This paper presented a novel hybrid segmentation algorithm that combines VFI and B-mode to delineate the vessel boundaries. The algorithm also implemented PSV and volume flow for VFI. The empirical results showed a dice coefficient of 0.91, indicating a good match between segmentation performed by the algorithm and the expert. The results of the flowrig measurements also showed that VFI was $15 \%$ more precise than spectral Doppler for PSV measurement. Therefore, the proposed method enables a better visualization of VFI as well as the automatic quantitative flow measures using vector velocities. The algorithm can also be used in daily clinical practice as an alternative tool for making a quantitative flow measures.

\section{REFERENCES}

[1] M. M. Pedersen, M. J. Pihl, P. Haugaard, J. M. Hansen, K. L. Hansen, M. B. Nielsen, and J. A. Jensen, "Comparison of real-time in vivo spectral and vector velocity estimation," Ultrasound Med. Biol., vol. 38, no. 1, pp. 145-151, 2012.

[2] E. G. Grant, C. B. Benson, G. L. Moneta, A. V. Alexandrov, J. D. Baker, E. I. Bluth, B. A. Carroll, and et al., "Carotid artery stenosis: gray-scale and doppler us diagnosis - society of radiologists in ultrasound consensus conference," Radiology, vol. 229, no. 2, pp. 340-346, 2003, m. Eliasziw and J. Gocke and B. S. Hertzberg.

[3] G. M. von Reutern, M. W. Goertler, N. M. Bornstein, M. D. Sette, D. H. Evans, A. Hetzel, M. Kaps, F. Perren, and et al., "Grading carotid stenosis using ultrasonic methods," Stroke, vol. 43, no. 3, pp. 916-921, 2012.

[4] P. M. Hansen, J. B. Olesen, M. J. Pihl, T. Lange, S. Heerwagen, M. M. Pedersen, M. Rix, L. Lønn, J. A. Jensen, and M. B. Nielsen, "Volume flow in arteriovenous fistulas using vector velocity ultrasound," Ultrasound Med. Biol., vol. 40, no. 11, pp. 2707-2714, 2014.

[5] A. H. Brandt, J. Jensen, K. L. Hansen, P. M. Hansen, T. Lange, M. Rix, J. A. Jensen, L. Lönn, and M. B. Nielsen, "Surveillance for hemodialysis access stenosis: Usefulness of ultrasound vector volume flow," J. Vascul. Access., p. Accepted for publication, 2016.

[6] J. Jensen, J. B. Olesen, P. M. Hansen, M. B. Nielsen, and J. A. Jensen, "Accuracy and sources of error for an angle independent volume flow estimator," Proc. IEEE Ultrason. Symp., pp. 1714-1717, 2014.

[7] P. M. Hansen, S. Heerwagen, M. M. Pedersen, M. Rix, L. Lønn, M. B. Nielsen, J. B. Olesen, M. J. Pihl, and J. A. Jensen, "Vector volume flow in arteriovenous fistulas," Proc. IEEE Ultrason. Symp., pp. 2026-2029, 2013.

[8] J. Jensen, J. B. Olesen, M. B. Stuart, P. M. Hansen, M. B. Nielsen, and J. A. Jensen, "Vector velocity volume flow estimation: Sources of error and corrections applied for arteriovenous fistulas," Ultrasonics, vol. 70, pp. 136-146, 2016. 